\title{
Performance Optimization of a Solar-Driven Multi-Step Irreversible Brayton Cycle Based on a Multi-Objective Genetic Algorithm
}

\author{
Mohammad Hosein Ahmadi ${ }^{*}$, Mohammad Ali Ahmadi ${ }^{2}$ and Michel Feidt ${ }^{3}$ \\ ${ }^{1}$ Department of Renewable Energies, Faculty of New Science and Technologies, University of Tehran, Tehran - Iran \\ 2 Department of Petroleum Engineering, Ahwaz Faculty of Petroleum Engineering, Petroleum University of Technology (PUT), Ahwaz - Iran \\ ${ }^{3}$ Laboratoire d'Énergétique et de Mécanique Théorique et Appliquée, ENSEM, 2 avenue de la Forêt de Haye, 54518 Vandœuvre - France \\ e-mail: mohammadhosein.ahmadi@gmail.com - ahmadi6776@yahoo.com - michel.feidt@univ-lorraine.fr \\ * Corresponding author
}

\begin{abstract}
An applicable approach for a multi-step regenerative irreversible Brayton cycle on the basis of thermodynamics and optimization of thermal efficiency and normalized outpuzt power is presented in this work. In the present study, thermodynamic analysis and a NSGA II algorithm are coupled to determine the optimum values of thermal efficiency and normalized power output for a Brayton cycle system. Moreover, three well-known decision-making methods are employed to indicate definite answers from the outputs gained from the aforementioned approach. Finally, with the aim of error analysis, the values of the average and maximum error of the results are also calculated.
\end{abstract}

Résumé - Optimisation des performances d'un cycle de Brayton irréversible solarisé à compressions et détentes multiples sur la base d'un algorithme génétique multi-objectif - Une approche applicable à un cycle de Brayton irréversible régénératif à compressions et détentes multiples, sur la base de la thermodynamique, de l'optimisation du rendement thermique et de la puissance de sortie normalisée, est présentée dans cet article. Dans la présente étude, l'analyse thermodynamique et un algorithme NSGA II sont couplés, afin de déterminer les valeurs optimales de rendement thermique et de puissance de sortie normalisée pour un système à cycle de Brayton. En outre, trois méthodes de prise de décision bien connues sont utilisées pour indiquer des réponses définies à partir des résultats obtenus à partir de l'approche mentionnée ci-dessus. Enfin, afin d'analyser les éventuelles erreurs, les valeurs de la moyenne et de l'erreur maximale des résultats sont également calculées. 


\section{NOMENCLATURE}

$A_{a} \quad$ Aperture area

$A_{r} \quad$ Absorber area

$a_{c} \quad$ Isentropic compressor pressure ratio

$C_{w} \quad$ Constant heat capacity rate of the working fluid

C Concentration ratio

$C_{i} \quad$ Internal conductance of the power plant

$C_{v} \quad$ Constant volume specific heat

$C_{i} \quad$ Decision index in TOPSIS approach

$d \quad$ Deviation index

$d_{i+} \quad$ Deviation or distance of $i$ th outcome from ideal outcome

$d_{i-} \quad$ Deviation or distance of $i$ th outcome from nonideal outcome

$G \quad$ The solar irradiance $\left(\mathrm{W} / \mathrm{m}^{2}\right)$

$P^{\prime} \quad$ The net output power of the cycle

$p \quad$ Pressure

$\Delta p \quad$ Pressure loss

$P \quad$ Normalized power

$\dot{Q} \quad$ Heat transfer rate

$N_{t} \quad$ Number of turbines

$N_{c} \quad$ Number of compressors

$r_{p} \quad$ Overall compression ratio

$T \quad$ Temperature

$U_{L} \quad$ Overall effective coefficient

$i \quad$ ith objective

$j \quad$ jth solution

$X \quad$ Vector of decision parameters

\section{Greek letter}

$\varepsilon \quad$ Irreversibilities

$\varepsilon_{r} \quad$ Effectiveness of the regenerator

$\varepsilon_{c} \quad$ Isentropic efficiency of the compressors

$\varepsilon_{L} \quad$ Isentropic efficiency of the turbines

$\varepsilon_{H} \quad$ Irreversibilities bring in the mixing of the working fluid with the heat reservoir at temperature $T_{H}$

$\varepsilon_{L} \quad$ Irreversibilities bring in the mixing of the working fluid with the heat reservoir at temperature $T_{L}$

$\eta \quad$ Efficiency

$\gamma \quad$ Adiabatic coefficient

$\mu \quad$ Fuzzy membership function

$\xi \quad$ Heat leakage throughout the plant to the surroundings

$\tau \quad$ Heat source temperature ratio

\section{Subscripts}

$H \quad$ Heat source

$L \quad$ Heat sink

1-4 The process states

\section{INTRODUCTION}

Nowadays, due to strategies minimizing the environmental difficulties and energy savings, solar-driven heat engines have attracted high attentions. Also, different heat engine cycles coupled with solar collectors have been investigated. In these researches, solar driven Carnot, Stirling, Ericsson and Braysson cycles were studied [1-16]. Academically, to enhance the performance of simple Brayton cycles, many modifications have been carried out to develop the system performance which consist of regeneration, isothermal heat addition, intercooler compression and reheat expansion [17-28]. Among the various objective functions that have been investigated for Brayton-based heat engines are power, efficiency [29-32], power density [33, 34], different ecological criteria [35, 36], thermoeconomic functions $[37,38]$ and other functions which have a compromise with output power and energy consumption [39]. In any case, as discussed by Chen [40] and AriasHernandez et al. [41] and Dong et al. [42], the optimal effectiveness of a heat engine is located between the maximum output power conditions and the maximum efficiency situation. As a special case, we ought to analyze the maximum efficiency regimes and maximum power of the given multi-step gas turbine.

The traditional single objective optimizations suffer from defect, since these optimize the systems based on only one criterion expressed by the objective functions. However, other aspects of design for perfect system planning are missed and those criteria might become worse than the previous optimizations. Therefore, attention to multi-objective optimizations has been paid by the majority of researchers in designing of thermal systems [43-55]. Firstly, Evolutionary Algorithms (EA) were evolved and employed in the previous decades to unravel random issues of this generic type [43]. A rational further step to a multi-objective issue is to assay a set of routes, which assures the objectives at a nice degree without being prevailed by any other routes [44]. Multi-objective optimization issues represent a great number of routes, and thus should be named as a Pareto frontier, whose assessed vectors illustrate the prior trade-offs' outcomes.

In the current work, a multi-step solar-driven Brayton engine was optimized using an evolutionary algorithm, 


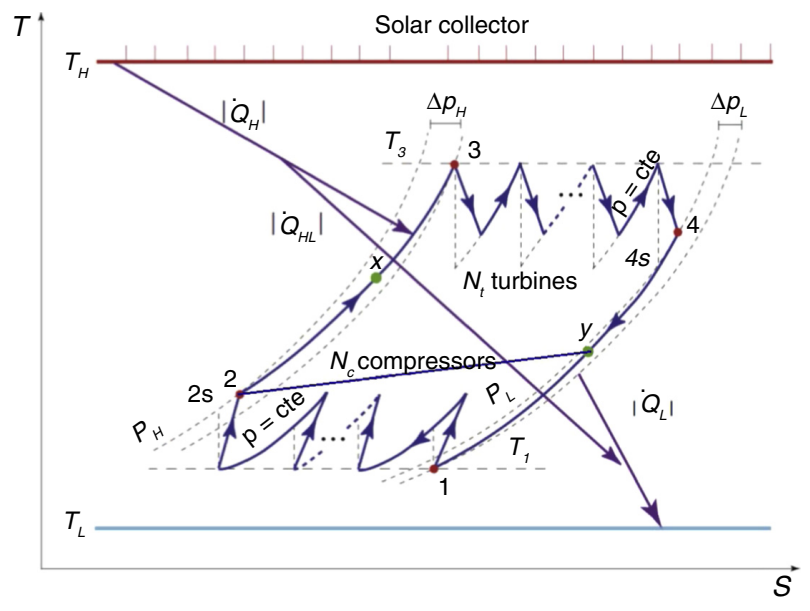

Figure 1

$T-S$ diagram of the thermodynamic cycle [56].

while thermal efficiency and the normalized output power in non-dimensional forms are presumed as the objectives of the optimization. For this solution, the operating parameters of thermal engine which includes the engine temperature ratio, irreversibilities (from coupling the working fluid with the heat source and heat sink), and regenerator effectiveness were considered as the decision variables.

\section{THEORETICAL MODEL}

The $T$-S diagram of the suggested approach and the layout carton for a Brayton heat engine with irreversible solar-driven multi-step are demonstrated in Figure 1, and the scheme of the plant which under studied here is depicted in Figure 2, [56]. Throughout the Brayton heat engine, from the solar collector at temperature $T_{H}$ it receives a net heat rate $\dot{Q}_{H}$ and asserts at temperature $T_{L}$ a net heat rate $\dot{Q}_{L}$ to the surroundings. Moreover, the linear behavior of heat leakage $\dot{Q}_{H L}$ regarding the heat source at $T_{H}$ to the heat sink at $T_{L}$ is presumed in the current study [6].

According to Duffie and Beckman [57] and Zhang et al. [58], a concentrating collector is considered where heat losses at intermediate and low temperatures are mainly affiliated with convection and conduction; however, at high sufficient temperatures, heat losses due to radiation are prevailing. Thanks to this consideration, in our approach, the effective energy attributed to the heat engine, $\dot{Q}_{H}$, and the solar collector's efficiency, $\eta_{s}$, can be formulated as following as [56, 58, 59]:

$$
\begin{aligned}
\dot{Q}_{H} & =\eta_{0} G A_{a}-U_{L} A_{r} T_{L}(\tau-1) \\
\eta_{s} & =\frac{\dot{Q}_{H}}{G A_{a}}=\eta_{0}(1-(\tau-1) M)
\end{aligned}
$$

In the aforementioned formulas, $\tau=T_{H} / T_{L}$ stands for the temperature ratio of the heat reservoirs; $G$ denotes the solar irradiance; $A_{r}$ and $A_{a}$ represent the absorber and aperture areas, correspondingly; $C=A_{a} / A_{r}$ denotes the concentration ratio; $\eta_{0}$ represents optical efficiency (the efficient transmittance absorptance product); and $M$ is formulated as $M=U_{L} T_{L} /\left(\eta_{0} G C\right)$, where $U_{L}$ stands for an accounted overall effective coefficient for losses due to radiation in terms of linear heat loss $[57,58]$. The fundamental formulations of the approach are as follows [30, 56, 58, 59]:

$$
\begin{aligned}
\dot{Q}_{H}= & C_{w} T_{L}\left(\varepsilon_{H}\left(\tau-Z_{c}\left(1-\varepsilon_{r}\right) \frac{T_{1}}{T_{L}}-\varepsilon_{r} Z_{t} \frac{T_{3}}{T_{L}}\right)\right. \\
& \left.+\varepsilon_{t}\left(N_{t}-1\right)\left(1-a_{t}^{\frac{-1}{N_{t}}}\right) \frac{T_{3}}{T_{L}}+\xi(\tau-1)\right)
\end{aligned}
$$

$$
\begin{aligned}
\dot{Q}_{L}= & C_{w} T_{L}\left(\varepsilon_{L}\left(-1-Z_{t}\left(1-\varepsilon_{r}\right) \frac{T_{3}}{T_{L}}+\varepsilon_{r} Z_{c} \frac{T_{1}}{T_{L}}\right)\right. \\
& \left.+\frac{1}{\varepsilon_{c}}\left(N_{c}-1\right)\left(a_{c}^{\frac{1}{T_{c}}}-1\right) \frac{T_{1}}{T_{L}}+\xi(\tau-1)\right)
\end{aligned}
$$$$
Z_{c}=1+\frac{a_{c}^{\frac{1}{N_{c}}}-1}{\varepsilon_{c}}
$$

$$
Z_{t}=1-\varepsilon_{t}\left(1-a_{t}^{\frac{-1}{N_{t}}}\right)
$$

$$
a_{t}=a_{c} \rho_{H} \rho_{L}
$$

$$
\begin{gathered}
\frac{T_{1}}{T_{L}}=\frac{\varepsilon_{L}+\left(1-\varepsilon_{L}\right)\left(1-\varepsilon_{r}\right) Z_{t} \frac{T_{3}}{T_{L}}}{1-\left(1-\varepsilon_{L}\right) \varepsilon_{r} Z_{c}} \\
\rho_{H}=\left(\frac{p_{3}}{p_{2}}\right)^{\frac{\gamma-1}{\gamma}}=\left(\frac{p_{H}-\Delta p_{H}}{p_{H}}\right)^{\frac{\gamma-1}{\gamma}} \\
\rho_{L}=\left(\frac{p_{1}}{p_{4}}\right)^{\frac{\gamma-1}{\gamma}}=\left(\frac{p_{L}-\Delta p_{L}}{p_{L}}\right)^{\frac{\gamma-1}{\gamma}}
\end{gathered}
$$

$$
\begin{aligned}
& \frac{T_{3}}{T_{L}}= \\
& \frac{\tau \varepsilon_{H}\left(1-\left(1-\varepsilon_{L}\right) \varepsilon_{r} Z_{c}\right)+\varepsilon_{L}\left(1-\varepsilon_{H}\right)\left(1-\varepsilon_{r}\right) Z_{c}}{\left(1-\left(1-\varepsilon_{L}\right) \varepsilon_{r} Z_{c}\right)\left(1-\left(1-\varepsilon_{H}\right) \varepsilon_{r} Z_{t}\right)-\left(1-\varepsilon_{H}\right)\left(1-\varepsilon_{L}\right)\left(1-\varepsilon_{r}\right)^{2} Z_{t} Z_{c}}
\end{aligned}
$$

where:

$C_{w}$ is the working fluid's constant heat capacity rate, 


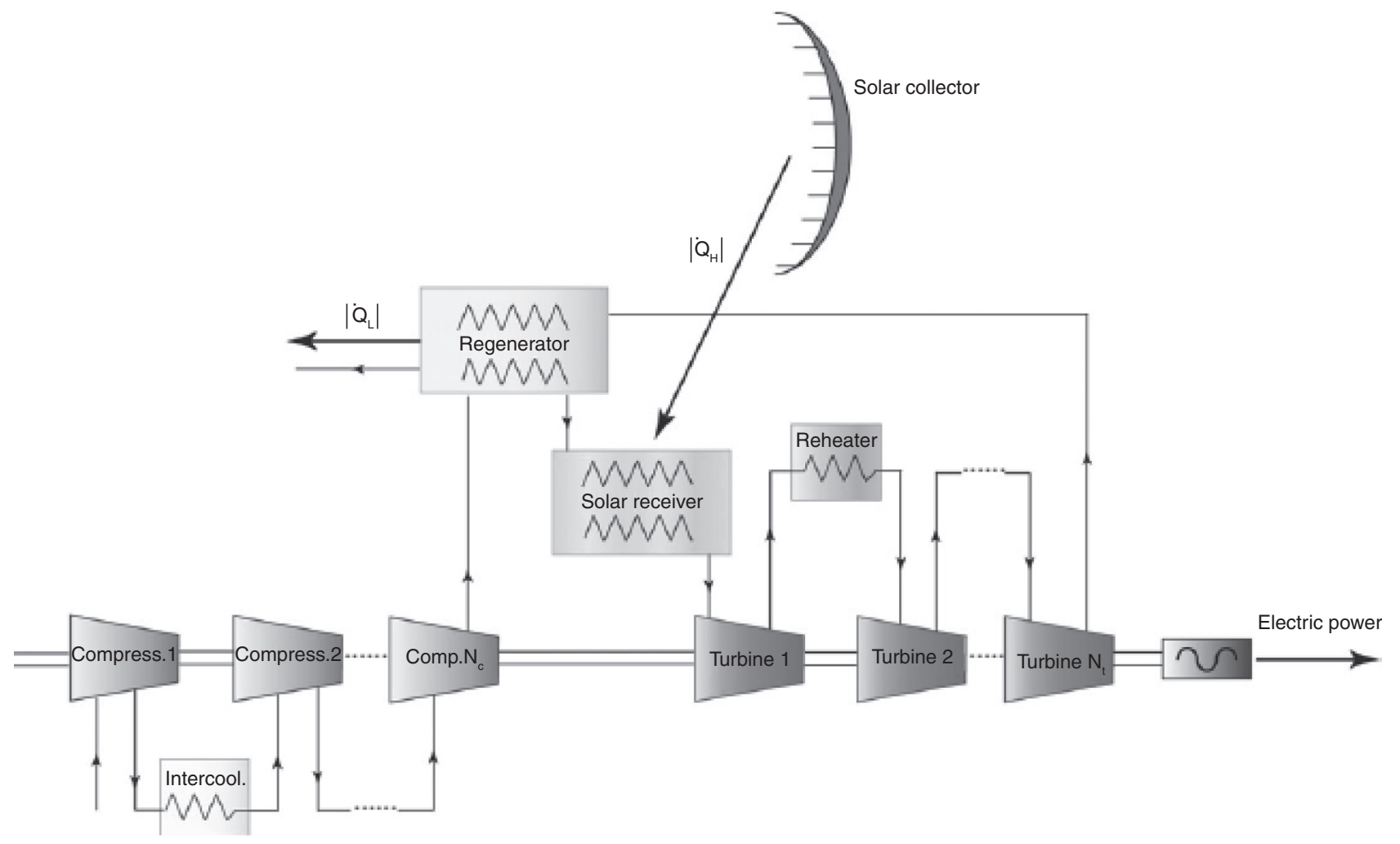

Figure 2

Scheme of the considered solar-driven Brayton plant [56].

$$
a_{c}=\frac{T_{2 s}}{T_{1}}=r_{p}^{\frac{\gamma-1}{\gamma}}
$$

is the pressure ratio of the isentropic compressor and $r_{p}=p_{2} / p_{1}$ represents the overall compression ratio. $\dot{Q}_{H L}=C_{i}\left(T_{H}-T_{L}\right)=C_{i} T_{L}(\tau-1)$ represents the leakage of heat during the heat reservoirs throughout the system. Furthermore, it can be formulated as $\xi(\tau-1)$, in which $\xi=C_{i} / C_{w}$ stands for the internal conductance ratio of the plant in terms of the working fluid and $C_{i}$ represents the power plant internal conductance $[6,35$, 56, 59]. Equations (3) and (4) express the Brayton heat engine efficiency for heat release and heat input, correspondingly

$$
\eta_{h}=\frac{P^{\prime}}{\dot{Q}_{H}}=\frac{\dot{Q}_{H}-\dot{Q}_{L}}{\dot{Q}_{H}}=1-\frac{\dot{Q}_{L}}{\dot{Q}_{H}}
$$

in which $P^{\prime}$ denotes the net output power of the cycle.

The overall solar-driven plant efficiency:

$$
\eta=\frac{P^{\prime}}{G A_{a}}
$$

can be formulated as the product of the effectiveness of the Brayton heat engine with multi-step and of the solar collector $[30,56,59]$ :

$$
\eta=\frac{P^{\prime}}{G A_{a}}=\frac{P^{\prime}}{\dot{Q}_{H}} \frac{\dot{Q}_{H}}{G A_{a}}=\eta_{s} \eta_{h}
$$

The normalized power is obtained by [30]:

$$
P=\frac{P^{\prime}}{C_{w} T_{L}}
$$

\section{MULTI-OBJECTIVE OPTIMIZATION WITH EVOLUTIONARY ALGORITHMS}

\subsection{Evolutionary Algorithms}

John Holland was a scientist who evolved and proposed a Genetic Algorithm (GA) in the 1960s inspired by natural adaption from Darwin's theory and imported it into a computer program for a stochastic optimization process [43]. Fundamentally, genetic algorithms employ 


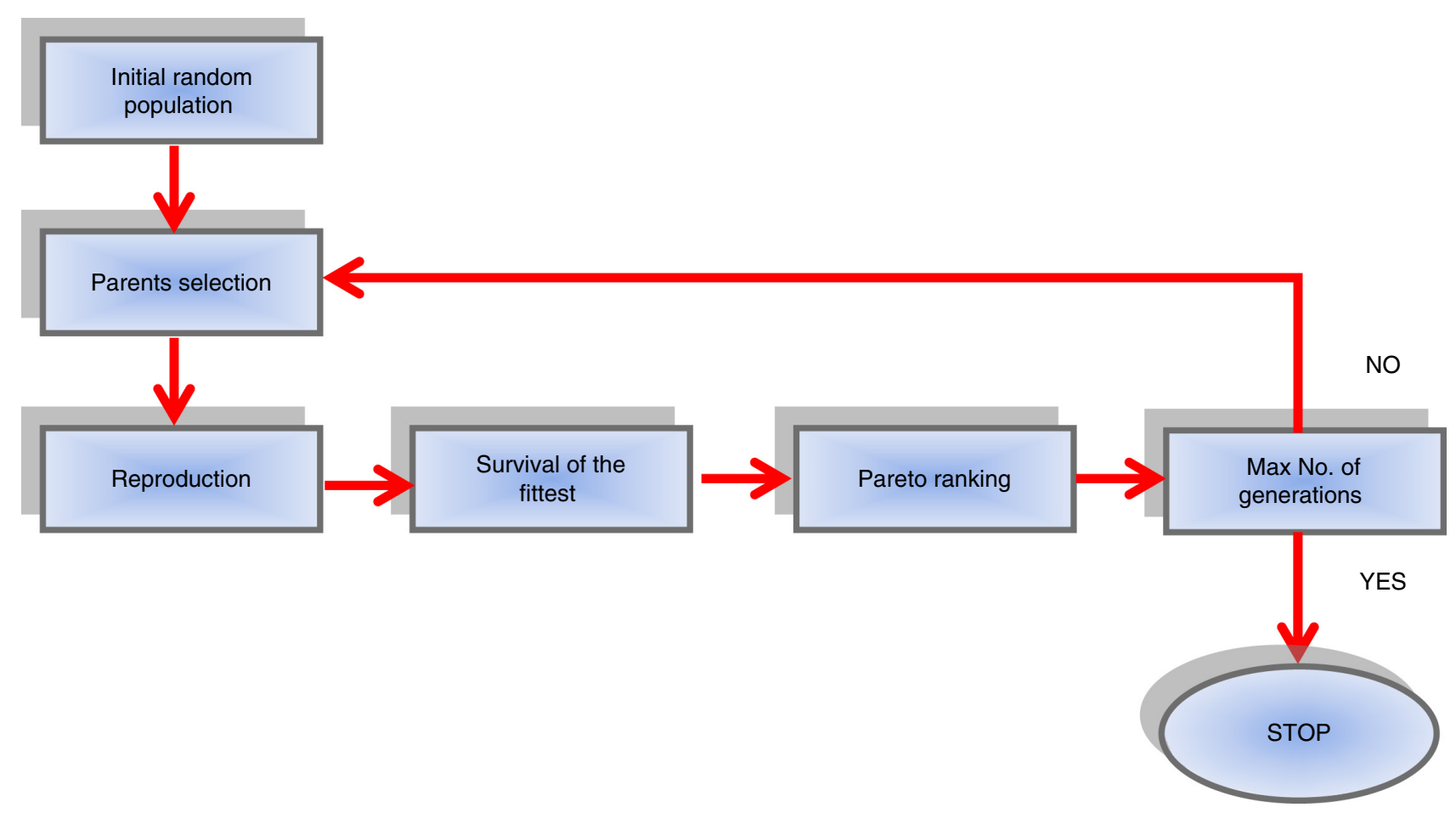

Figure 3

Scheme for the multi-objective evolutionary algorithm used in the present study $[46,47,60]$.

stochastic and iterative search strategy to determine an optimum route and mimic a reduced maxim of biological evolution (Fig. 3). In the current research, a GA as a split of evolutionary algorithm is utilized to determine the Pareto frontier. As noted previously, GA is a robust and effective tool for optimization of nonlinear issues $[44,45]$. The optimality of Pareto is an important sense to specify a ranking between the routes of a multiobjective optimization issue, in order to indicate which solution is prior to other possible solutions [45]. In this communication, a NSGA-II approach is executed as a multi-objective optimization algorithm to figure out the Pareto frontier by utilizing a GA [45, 48, 60-62].

\subsection{Objective Functions, Decision Variables and Constraints}

Two objective functions for the current research are thermal efficiency $(\eta)$ and the normalized power output $(P)$, which are denoted by Equations $(13,14)$, correspondingly.

Throughout this research, four decision variables including temperature ratio $\left(x=T_{3} / T_{L}\right)$, engine temperature ratio $(\tau)$, irreversibilities $(\varepsilon)$ and regenerator effectiveness $\left(\varepsilon_{r}\right)$ were considered. The objective functions are optimized regard to the below restrictions on the decision variables:

$$
\begin{gathered}
2.25 \leq \frac{T_{3}}{T_{L}} \leq 3 \\
3 \leq \tau \leq 4 \\
0.7 \leq \varepsilon \leq 0.9 \\
0.8 \leq \varepsilon_{r} \leq 1
\end{gathered}
$$

\section{DECISION-MAKING IN THE MULTI-OBJECTIVE OPTIMIZATION}

Throughout multi-objective subjects, the algorithm of decision-making to elect the best route from among other possible outcomes is needed. Many procedures can be applied to make decision for taking the ultimate route from the Pareto frontier. In the first step, the scales and dimensions of the objective region have to be incorporated thanks to the eventuality of various dimensions that may exist in the multi-objective optimization 


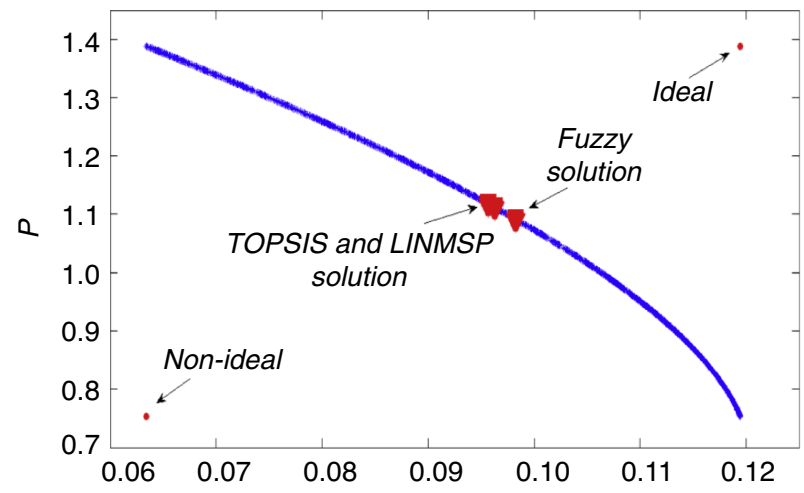

a)

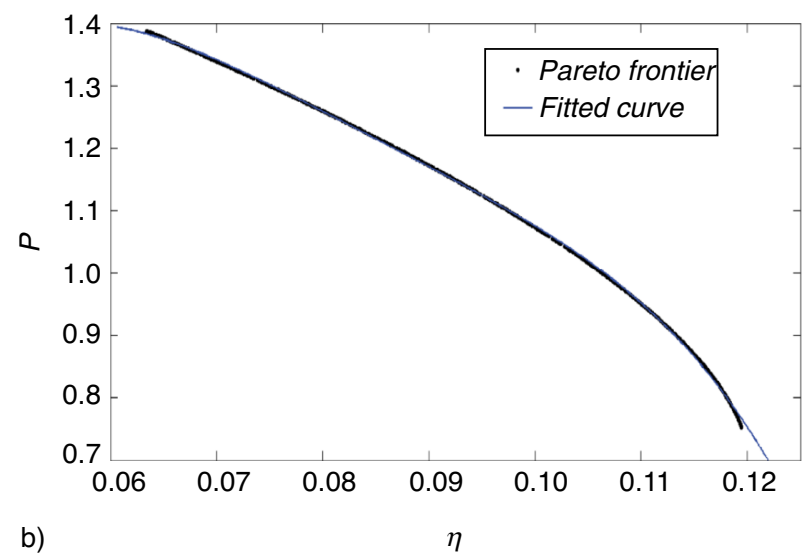

Figure 4

a) The original Pareto optimal curve; b) the optimal curve with curve fit.

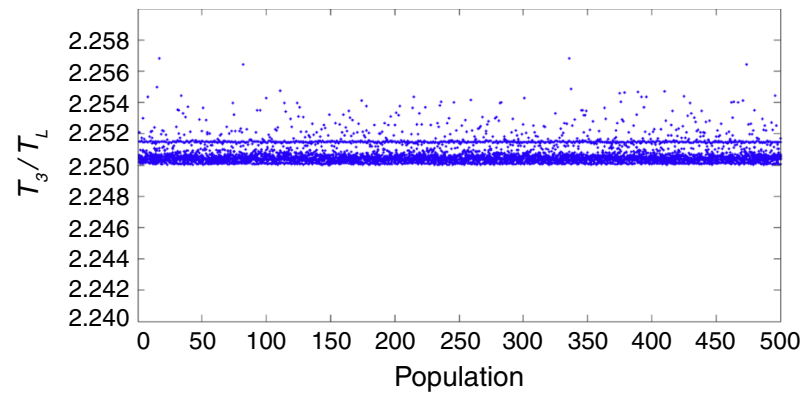

Figure 5

Scatter distribution of $T_{3} / T_{L}$ with population in Pareto frontier.

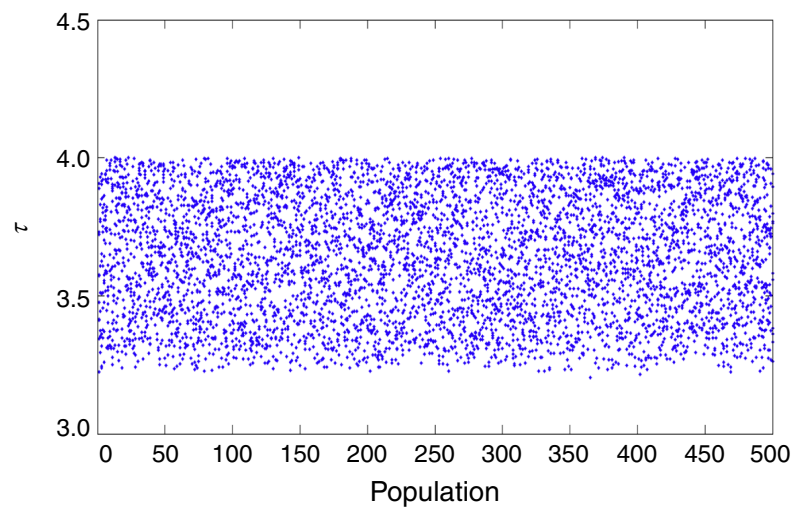

Figure 6

Scatter distribution of $\tau$ with population in Pareto frontier. process. In fact, the objective vectors have to be dimensionless prior to the decision-making algorithm. Different approaches to dimensionless utilization in decision-making include linear, Euclidean and fuzzy. Detailed descriptions regarding these methods of nondimensioning were reported in [48]. In addition, more details of the decision-making approaches addressed are found in the literature, such as $[46-49,60]$.

\section{RESULTS AND DISCUSSION}

The thermal efficiency and the normalized output power of an irreversible Brayton regenerative cycle were maximized. These objectives are optimized concurrently executing a type of multi-objective evolutionary algorithms called NSGA-II. The process of optimization is performed by executing the objective functions expressed by Equations $(13,14)$ beside the limitations which are stated in Equations (15-18). Figure 4a depicts the Pareto frontier in the presumed objectives' region that gained from the scenario. Moreover, optimum solutions which are gained by performing LINMAP, Fuzzy Bellman-Zadeh, and TOPSIS decision-makers are demonstrated in the aforementioned figure. According to Figure $4 \mathrm{a}$, the points obtained by LINMAP and TOPSIS have an approaching trend. Also it is shown that the optimal value of $P$ varies from 0.75 to 1.4 and the optimal value of $\eta$ is 0.0625 to 0.12 . Based on the curve fit obtained depicted in Figure 4b, Equation (19) was developed:

$$
P=p_{1} \eta^{4}+p_{2} \eta^{3}+p_{3} \eta^{2}+p_{4} \eta+p_{5}
$$

Where $\eta$ is normalized by mean 0.09689 and std 0.01717 coefficients (with 95\% confidence bounds):
$p_{1}=-0.008249$
$p_{2}=-0.02302$
$p_{3}=-0.026$
$p_{4}=-0.166$
$p_{5}=1.106$ 


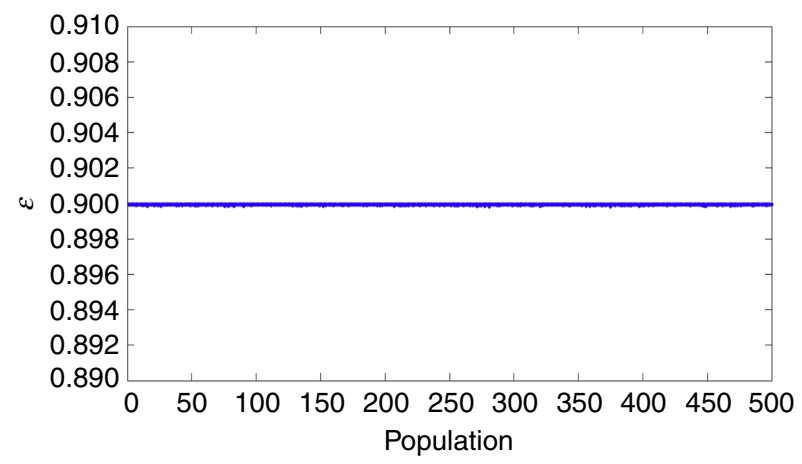

Figure 7

Scatter distribution of $\varepsilon$ with population in Pareto frontier.

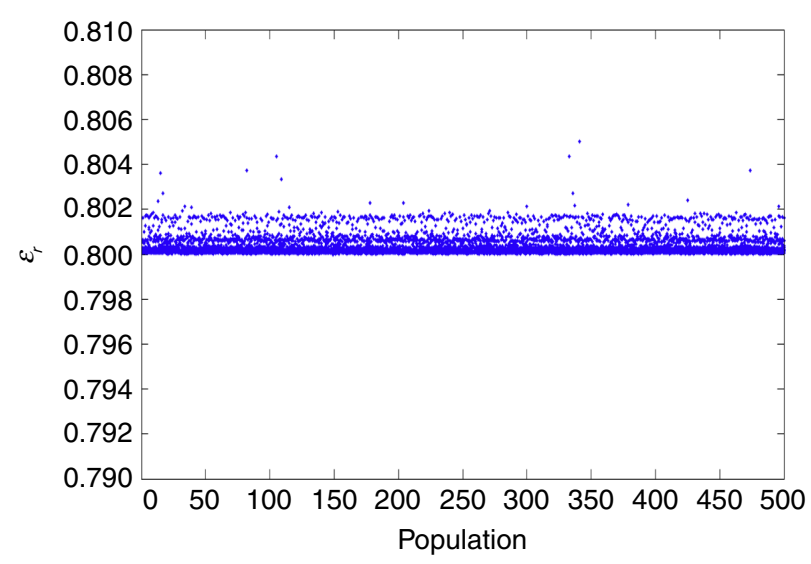

Figure 8

Scatter distribution of $\varepsilon_{r}$ with population in Pareto frontier.

Goodness of fit:

SSE: 0.007218

$R^{2}: 0.9997$

RMSE: 0.003013

Figures 5-8 demonstrate the scattered distribution of the design variables versus the population. These figures can provide a better vision of the variation of the decision variables from the Pareto frontier. According to this distribution, it is found that the irreversibilities bring in the mixing of the working fluid with the heat sink and the heat source meets their maximum magnitudes. It displays that increasing the design parameters results in enhancement in both objective functions. Figure 5 depicts the range of variation of $x=T_{3} / T_{L}$ for an optimal Pareto front. The distribution of variables for the optimum points on the Pareto front for $\tau$ over the population are exhibited in Figure 6 and the range of $\tau$ varied from 3.25 to 4 . It can be seen from Figure 7 that the magnitude of $\varepsilon$ for an optimal Pareto front is equal to 0.9. As shown in Figure 8, the changes in $\varepsilon_{r}$ for an optimal Pareto front varied from 0.8 to 0.802 .

The optimum outcomes for decision variables and objective functions executing TOPSIS, LINMAP and Bellman-Zadeh decision-making approaches are explained in Table 1. Table 1 demonstrates results gained from the aforementioned decision-makers for the solar-driven multi-step irreversible Brayton cycle and outcomes described by Sánchez-Orgaz et al. $[56,59]$. For better grasp regarding the situation of different outcomes gained here in contrast with the outcome of the single objective-single variable approach $[56,59]$, a deviation index which determines the deviation of each outcome from the ideal outcome is introduced here:

$$
\begin{gathered}
d_{+}=\sqrt{\left(p_{n}-p_{n, \text { ideal }}\right)^{2}+\left(\eta-\eta_{n, \text { ideal }}\right)^{2}} \\
d_{-}=\sqrt{\left(p_{n}-p_{n, \text { non-ideal }}\right)^{2}+\left(\eta-\eta_{n, \text { non-ideal }}\right)^{2}} \\
d=\frac{d_{+}}{\left(d_{+}\right)+\left(d_{-}\right)}
\end{gathered}
$$

$p_{n}$ and $\eta_{n}$ stand for Euclidian non-dimensionalized normalized output power and thermal efficiency (the indices ideal and non-ideal denote the relevant amounts of objective functions at the ideal and non-ideal data points, correspondingly). The last column of Table 1 denotes the deviation index $(d)$ for the outcomes gained from each decision-makers. As illustrated in Table 1, the deviation indices of the multi-objective optimization method throughout the current study are $0.4159,0.4253$ and 0.4562 for outcomes that were gained by utilizing the FUZZY, LINMAP and TOPSIS decision-makers, correspondingly. These values are analogous with the relevant value gained previously by performing the single objective-single variable optimization approach [56, 59] where the deviation index is 0.4785 . This indicates that the deviation indices of the single objective-single variable optimization are greater than the multi-objectivemulti-variable optimization for all decision-makers while the ideal deviation index is zero.

Further, the last column of Table 1 indicates that the FUZZY decision-maker leads to a lower value for deviation index; therefore, throughout the current study, the outcome that is chosen by executing the FUZZY decision-maker is presumed to be an ultimate optimum outcome of the multi-objective-multi-parameter optimization process for the solar-driven multi-step irreversible 
TABLE 1

Decision-making of multi-objective optimal solutions

\begin{tabular}{c|c|c|c|c|c|c|c}
\hline \multirow{2}{*}{$\begin{array}{c}\text { Decision- } \\
\text { making method }\end{array}$} & \multicolumn{4}{|c|}{ Decision variables } & \multicolumn{2}{c|}{ Objective functions } & $\begin{array}{c}\text { Deviation index from the } \\
\text { ideal solution }(d)\end{array}$ \\
\cline { 2 - 8 } & $\tau$ & $\frac{T_{3}}{T_{L}}$ & $\varepsilon_{r}$ & $\varepsilon$ & $\eta$ & $P$ & 0.4159 \\
\hline Fuzzy & 3.695164 & 2.250287 & 0.8 & 0.9 & 0.095673 & 1.11791522 & 0.4253 \\
\hline LINMAP & 3.68845 & 2.250166 & 0.8 & 0.9 & 0.096280 & 1.11194254 & 0.4562 \\
\hline TOPSIS & 3.666383 & 2.250066 & 0.8 & 0.9 & 0.098223 & 1.09212691 & 0.4785 \\
\hline $\begin{array}{c}\text { References } \\
{[56,59]}\end{array}$ & 3.6 & - & 0.8 & 0.9 & 0.100000 & 0.80000000 & \\
\hline Ideal point & - & - & - & - & 0.118000 & 1.38000000 & 0.0000 \\
\hline Non-ideal point & - & - & - & - & 0.063000 & 0.75000000 & $\infty$ \\
\hline
\end{tabular}

TABLE 2

Error analysis based on the Mean Absolute Percent Error (MAPE) method

\begin{tabular}{c|c|c|c|c|c|c}
\hline $\begin{array}{c}\text { Decision-making } \\
\text { method }\end{array}$ & \multicolumn{2}{|c|}{ LINMAP } & \multicolumn{2}{c}{ TOPSIS } & $P$ & \\
\hline Objectives & $\eta$ & $P$ & $\eta$ & 2.06 & 3.18 & $P$ \\
\hline Max error (\%) & 1.28 & 1.16 & 2.26 & 0.90 & 1.46 & 2.94 \\
\hline $\begin{array}{c}\text { Average error } \\
(\%)\end{array}$ & 0.55 & 0.46 & 1.03 & & 1.35 \\
\hline
\end{tabular}

Brayton cycle. It is worth bearing in mind that this is not a universal approach for other cases, so other decisionmakers might have superior outcomes.

\section{ERROR ANALYSIS}

To compare each of the decision-makers and select the most robust one, the Mean Absolute Percentage Error (MAPE) is utilized to indicate the amount of error for the aforementioned decision-makers. To this end, each approach was executed 30 times to gain the ultimate outcome by LINMAP, Bellman-Zadeh and TOPSIS decision-makers. The first rank throughout Table 2 exhibits the Maximum Absolute Average Error (MAAE) of each decision-maker. Besides, the second row of Table 2 reports the MAPE of the decision-makers executed in our study.

\section{CONCLUSIONS}

In this study, an analytical model for an irreversible regenerative multi-step Brayton cycle was applied to determine the normalized power output $(P)$ and the thermal efficiency $(\eta)$ of the cycle. The thermal efficiency $(\eta)$ and the normalized power output $(P)$ of the multi-step irreversible Brayton cycle are spotted in parallel throughout the multi-objective optimization process. The heat source to heat sink and regenerator effectiveness $\left(\varepsilon_{r}\right)$, temperature ratio $(x)$, engine temperature ratio $(\tau)$, and irreversibilities $(\varepsilon)$ are presumed to be the design variables. NSGA-II is exploited throughout the multi-criteria optimization process and further, the Pareto optimal frontier throughout the objective space is determined. By performing three rigorous decision-making methods, three different optimum solutions are gained, regarding which the decision making approach was executed. By taking plots of previous works into account, the optimum results gained by the aforementioned decision-making approaches TOPSIS, LINMAP and Fuzzy were compared with a relevant actual one on the basis of the deviations from the ideal state. As can clearly be seen, the lowest deviation in the results refers to the Fuzzy method, which is superior in contrast to the ideal; conversely, greater deviation from the ideal state refers to previous research. These comparisons prove that the results of this optimization are acceptable. 


\section{REFERENCES}

1 Gordon J.M. (1988) On optimized solar-driven heat engines, Solar Energy 40, 457-461.

2 Sahin A.Z. (2000) Optimum operating conditions of solardriven heat engines, Energy Conversion and Management 41, 1335-1343.

3 Koyun A. (2004) Performance analysis of a solar-driven heat engine with external irreversibilities under maximum power and power density condition, Energy Conversion and Management 45, 1941-1947.

4 Sogut O.S., Durmayaz A. (2005) Performance optimization of a solar-driven heat engine with finite-rate heat transfer, Renewable Energy 30, 1329-1344.

5 Yilmaz T., Ust Y., Erdil A. (2006) Optimum operating conditions of irreversible solar driven heat engines, Renewable Energy 31, 1333-1342.

6 Ust Y. (2007) Effects of combined heat transfer on the thermoeconomic performance of irreversible solar-driven heat engines, Renewable Energy 32, 2085-2095.

7 Barranco-Jiménez M.A., Sánchez-Salas N. (2008) On thermodynamic optimization of solar collector model under maximum ecological conditions, Journal of the Energy Institute 81, 164-167.

8 Blank D.A., Wu C. (1998) Finite-time power limit for solarradiant Ericsson engines in space applications, Applied Thermal Engineering 18, 1347-1357.

9 Bädescu V. (1992) Optimum operation of a solar converter in combination with a Stirling or Ericsson heat engine, Energy 17, 601-607.

10 Ahmadi M.H., Hosseinzade H., Sayyaadi H., Mohammadi A.H., Kimiaghalam F. (2013) Application of the multiobjective optimization method for designing a powered Stirling heat engine: design with maximized power, thermal efficiency and minimized pressure loss, Renewable Energy 60, 313-322.

11 Ahmadi M.H., Sayyaadi H., Dehghani S., Hosseinzade H. (2013) Designing a solar powered Stirling heat engine based on multiple criteria: maximized thermal efficiency and power, Energy Conversion and Management 75, 282-291.

12 Ahmadi M.H., Mohammadi A.H., Dehghani S., BarrancoJiménez M.A. (2013) Multi-objective thermodynamicbased optimization of output power of solar dish-Stirling engine by implementing an evolutionary algorithm, Energy Conversion and Management 75, 438-445.

13 Chen J., Yan Z., Chen L., Andresen B. (1998) Efficiency bound of a solar driven Stirling heat engine system, International Journal of Energy Research 22, 805-812.

14 Zhang Y., Lin B., Chen J. (2005) The unified cycle model of aclass of solar-driven heat engines and their optimum performance characteristics, Journal of Applied Physics 97, 8490-8495.

15 Zheng S., Chen J., Lin G. (2005) Performance characteristics of an irreversible solar-driven Braysson heat engine at maximum efficiency, Renewable Energy 30, 601-610.

16 Wu L., Lin G., Chen J. (2010) Parametric optimization of a solar-driven Braysson heat engine with variable heat capacity of the working fluid and radiation-convection heat losses, Renewable Energy 35, 95-100.

17 Durmayaz A., Sogut O.S., Sahin B., Yamuz H. (2004) Optimization of thermal systems based on finite-time thermodynamics and thermo economics, Prog. Energy Combust. Sci. 30, 175-217.
18 Cheng C.Y., Chen C.K. (1996) Power optimization of an endoreversible regenerative Brayton cycle, Energy 21, 241-247.

19 Chen L.G., Sun F., Wu C., Kiang R.L. (1997) Theoretical analysis of the performance of our generative closed Brayton cycle with internal irreversibilities, Energy Conversion and Management 3, 871-877.

20 Medina A., Roco J.M.M., Calvo Hernández A. (1996) Regenerative gas turbines at maximum power density conditions, J. Phys. D: Appl. Phys. 29, 2802-2805.

21 Götkun S., Yavuz H. (1999) Thermal efficiency of a regenerative Brayton cycle with isothermal heat addition, Energy Conversion and Management 40, 1259-1266.

22 Erbay L., Götkun S., Yarvuz H. (2001) Optimal design of the regenerative gas turbine engine with isothermal heat addition, Appl. Energy 6, 249-264.

23 Vecchiarelli J., Kawall J.G., Wallace J.S. (1997) Analysis of a concept for increasing the efficiency of a Brayton cycle via isothermal heat addition, Int. J. Energy Res. 2, 113-127.

24 Cheng C.Y., Chen C.K. (2000) Maximum power of an endo reversible intercooled Brayton cycle, Int. J. Energy Res. 24, 485-494.

25 Wang W., Chen L., Sun F., Wu C. (2003) Performance analysis of an irreversible variable temperature heat reservoir closed intercooled regenerated Brayton cycle, Energy Conversion and Management 44, 2713-2732.

26 Calvo Hernández A., Roco J.M.M., Medina A. (1996) Power and efficiency in a regenerative gas-turbine with multiple reheating and intercooling stages, J. Phys. D: Appl. Phys. 29, 1462-1468.

27 Sogut O.S., Ust Y., Sahin B. (2006) The effects of intercooling and regeneration on the thermo-ecological performance analysis of an irreversible-closed Brayton heat engine with variable temperature thermal reservoirs, J. Phys. D: Appl. Phys. 39, 4713-4721.

28 Tyagi J.K., Chen G.M., Wang Q., Kaushik S.C. (2006) Thermodynamic analysis and parametric study of an irreversible regenerative-intercooled-reheat Brayton cycle, Int. J. Therm. Sci. 45, 829-840.

29 Calvo Hernández A., Medina A., Roco J.M.M. (1995) Power and efficiency in a regenerative gas turbine, J. Phys. D: Appl. Phys. 28, 2020-2023.

30 Sánchez-Orgaz S., Medina A., Calvo Hernández A. (2010) Thermodynamic model and optimization of a multi-step irreversible Brayton cycle, Energy Conversion and Management 51, 2134-2143.

31 Bejan A. (1988) Theory of heat transfer irreversible power plants, Int. J. Heat Mass Transfer 31, 1222-1229.

32 Landsberg P.T., Leff H.S. (1989) Thermodynamic cycles with nearly universal maximum-work efficiencies, J. Phys. A 22, 4019-4026.

33 Chen L., Zeng J., Sun F., Wu C. (2001) Power density analysis and optimization of a regenerated closed variabletemperature heat reservoir Brayton cycle, J. Phys. D: Appl. Phys. 34, 1727-1739.

34 Sahin B., Kodal A., Kaya S.S. (1998) A comparative performance analysis of irreversible reheating Joule-Brayton engines under maximum power density and maximum power conditions, J. Phys. D: Appl. Phys. 31, 2125-2131. 
35 Ust Y., Sahin B., Kodal A., Akcay I.H. (2006) Ecological coefficient of performance analysis and optimization of an irreversible regenerative-Brayton heat engine, Appl. Energy 83, 558-572.

36 Angulo-Brown F. (1991) An ecological optimization criterion for finite-time heat engines, J. Appl. Phys. 69, 7465-7469.

37 Sieniutycz S., Salamon P. (eds) (1990) Finite-time thermodynamics and thermo-economics, Taylor \& Francis.

38 Valero A., Correas L., Serra L. (1999) Thermodynamic optimization of complex energy systems, Kluwer, p. 117.

39 Calvo Hernández A., Medina A., Roco J.M.M., White J. A., Velasco S. (2001) Unified optimization criterion for energy converters, Phys. Rev. E. 63, 0371021-0371023.

40 Chen J. (1994) The maximum power output and maximum efficiency of an irreversible Carnot engine, J. Phys. D: Appl. Phys. 27, 1144-1149.

41 Arias-Hernandez L.A., Barranco-Jiménez M.A., AnguloBrown F. (2009) Comparative analysis of two ecological type modes of performance for a simple energy converter, J. Energy Inst. 82, 223-227.

42 Dong Y., El-Bakkali A., Feidt M., Descombes G., Périlhon C. (2012) Association of Finite-Dimension Thermodynamics and a Bond-Graph Approach for Modeling an Irreversible Heat Engine, Entropy 14, 1234-1258.

43 Holland J.H. (1975) Adaptation in Natural and Artificial Systems, University of Michigan Press, Ann Arbor.

44 Salcedo R., Antipova E., Boer D., Jiménez L., GuillénGosálbez G. (2012) Multi-objective optimization of solar Rankine cycles coupled with reverse osmosis desalination considering economic and life cycle environmental concerns, Desalination 286, 358-371.

45 Moura P.S., de Almeida A.T. (2010) Multi-objective optimization of a mixed renewable system with demand-side management, Renewable and Sustainable Energy Reviews 14, 1461-1468.

46 Ahmadi M.H., Mohammadi A.H., Dehghani S., Feidt M., Barranco-Jiménez M.A. (2013) Optimal design of a solar driven heat engine based on thermal and thermo-economic criteria, Energy Conversion and Management 75, 635-642.

47 Ahmadi M.H., Mohammadi A.H., Dehghani S. (2013) Evaluation of the maximized power of a regenerative endoreversible Stirling cycle using the thermodynamic analysis, Energy Conversion and Management 76, 561-570.

48 Mohammad H. Ahmadi, Amir H. Mohammadi, S. Mohsen Pourkiaei (2014): Optimization of the thermodynamic performance of the Stirling engine, International Journal of Ambient Energy, DOI:10.80/01430750.2014.907211.

49 Lazzaretto A., Toffolo A. (2004) Energy, economy and environment as objectives in multi-criterion optimization of thermal systems design, Energy 29, 1139-1157.
50 Toffolo A., Lazzaretto A. (2002) Evolutionary algorithms for multi-objective energetic and economic optimization in thermal system design, Energy 27, 549-567.

51 Dhanalakshmi S., Kannan S., Mahadevan K., Baskar S. (2011) Application of modified NSGA-II algorithm to combined economic and emission dispatch problem, Int. J. Electr. Power Energy Syst. 33, 4, 992-1002.

52 Pires D.F., Antunes C.H., Martins A.G. (2012) NSGA-II with local search for a multi-objective reactive power compensation problem, Int. J. Electr. Power Energy Syst. 43, 1, 313-324.

53 Hoseyn Sayyaadi, Mohammad Hossein Ahmadi, Saeed Dehghani (2014). Optimal Design of a Solar-Driven Heat Engine Based on Thermal and Ecological Criteria. J. Energy Eng., 10.1061/(ASCE)EY.1943-7897.0000191, 04014012 .

54 Najafi H., Najafi B. (2010) Multi-objective optimization of a plate and frame heat exchanger via genetic algorithm, Heat Mass Transfer 46, 639-647.

55 Ahmadi M.H., Ahmadi M.A., Mohammadi A.H., Feidt M., Pourkiaei S.M. (2014) Multi-objective optimization of an irreversible Stirling cryogenic refrigerator cycle, Energy Conversion and Management 82, 351-360.

56 Sánchez-Orgaz S., Medina A., Calvo Hernández A. (2013) Recuperative solar-driven multi-step gas turbine power plants, Energy Conversion and Management 67, 171-178.

57 Duffie J.A., Beckman W.A. (2006) Solar Engineering of Thermal Processes, John Wiley and Sons, Hoboken, New Jersey.

58 Zhang Y., Lin B., Chen J. (2007) Optimum performance characteristics of an irreversible solar-driven Brayton heat engine at the maximum overall efficiency, Renewable Energy 32, 856-867.

59 Sánchez-Orgaz S., Medina A., Calvo Hernández A. (2012) Maximum overall efficiency for a solar-driven gas turbine power plants, Int. J. Energy Res. 37, 13, 1580-1591.

60 Ahmadi M.H., Ahmadi M.A., Mohammadi A.H., Mehrpooya M., Feidt M. (2014) Thermodynamic optimization of Stirling heat pump based on multiple criteria, Energy Conversion and Management 80, 319-328.

61 Toghyani S., Kasaeian A., Ahmadi M.H. (2014) Multi-objective optimization of Stirling engine using non-ideal adiabatic method, Energy Conversion and Management 80, 54-62.

62 Taal M., Bulatov I., Klemeš J., Stehlik P. (2003) Cost estimation and energy price forecasts for economic evaluation of retrofit projects, Applied Thermal Engineering 23, 1819-1835.

Manuscript accepted in May 2014 Published online in September 2014

Cite this article as: M.H. Ahmadi, M.A. Ahmadi and M. Feidt (2014). Performance Optimization of a Solar-Driven Multi-Step Irreversible Brayton Cycle Based on a Multi-Objective Genetic Algorithm, Oil Gas Sci. Technol 71, 16. 


\section{APPENDIX: DECISION-MAKING IN THE MULTI-OBJECTIVE OPTIMIZATION}

To obtain the optimum value of the design parameters during multi-objective optimization in regard to satisfying the termination conditions, decision-making is used. Put another way, decision-making is executed to determine the best solution among all of the other possible solutions. Owing to this fact, different methods and algorithms can be used to make a decision whose solution from the Pareto frontier is better or not. Before tackling the methods of decisionmaking that was utilized in our study in detail, the scales and dimensions of the objective region have to be incorporated thanks to the eventuality of various dimensions that may exist in the multi-objective optimization process. Meanwhile, the vectors of objectives have to be non-dimensioned prior to making a decision. For this end, approaches such as fuzzy, Euclidean and linear non-dimensioning can be carried out throughout the process of decision-making. More explanation is given in the work performed by Ahmadi et al. [10].

\section{- Linear non-dimensionalization}

$F_{i j}$ represents the matrix of objectives at different data points of the Pareto frontier in which $i$ stands for the index of each aforementioned point on the Pareto frontier and $j$ denotes the index of each objective throughout the space of objectives. Accordingly, a non-dimensionalized objective, $F_{i j}^{n}$, can be expressed as follows:

$$
\begin{gathered}
F_{i j}^{n}=\frac{F_{i j}}{\max \left(F_{i j}\right)} \text { Maximizing orientation } \\
F_{i j}^{n}=\frac{F_{i j}}{\max \left(1 / F_{i j}\right)} \text { Minimalizing orientation }
\end{gathered}
$$

\section{- Euclidian non-dimensionalization}

Throughout this approach, a non-dimensionalized objective, $F_{i j}^{n}$, is formulated as below:

$$
F_{i j}^{n}=\frac{F_{i j}}{\sqrt{\sum_{i=1}^{m}\left(F_{i j}\right)^{2}}} \text { Minimizing and maximizing orientations }
$$

\section{- Fuzzy non-dimensionalization}

Throughout this approach, a non-dimensionalized objective, $F_{i j}^{n}$, is formulated as below [10-12]:

$$
\begin{aligned}
F_{i j}^{n} & =\frac{F_{i j}-\min \left(F_{i j}\right)}{\max \left(F_{i j}\right)-\min \left(F_{i j}\right)} \text { Maximizing orientations } \\
F_{i j}^{n} & =\frac{\max \left(F_{i j}\right)-F_{i j}}{\max \left(F_{i j}\right)-\min \left(F_{i j}\right)} \text { Minimizing orientation }
\end{aligned}
$$

In the current research, the rigorous and efficient types of decision-making algorithms that include TOPSIS, LINMAP and the fuzzy Bellman-Zadeh approach are utilized in order to compare their robustness toward obtaining the most reliable solutions. It is worth bearing in mind that determining the optimum solution from the final optimum solutions which were gained by the aforementioned decision-making methods are on the basis of engineering experience and criteria suggested previously. The Bellman-Zadeh approach carries out fuzzy non-dimensionalization, whereas the LINMAP and TOPSIS approaches utilize Euclidian non-dimensionalization. The next sections demonstrate the details of the above-mentioned algorithms for making decisions through the optimization process.

\section{A.1. Bellman-Zadeh's Decision-Making Method}

According to an approach evolved by Bellman and Zadeh (1970), the fuzzy set or fuzzy objective function should be replaced by $F_{j}(x)$ :

$$
A_{j}=\left\{X, \mu_{A_{j}}(X)\right\} \quad X \in L, j=1,2, \ldots, k
$$


In which $\mu_{A_{j}}(X)$ denotes $A_{j}$ 's membership function [10].

The junction between all of the fuzzy criteria and restrictions in the Bellman and Zadeh approach represents the final decision; meanwhile, it is presented by means of membership function. A fuzzy route D establishing the fuzzy assortments (Eq. 5) is procreated as an outcome of the conjunction $D=\cap_{j=1}^{k} A_{j}$ with a membership function:

$$
\mu_{D}(X)=\cap_{j=1}^{k} \mu_{A_{j}}(X)=\min _{j=1, \ldots, k} \mu_{A_{j}}(x), \quad X \in L
$$

By utilizing Equation (3a), it is feasible to gain the outcome verifying the maximum level as follows:

$$
\begin{gathered}
\max \mu_{D}(X)=\max _{X \in L} \min _{j=1, \ldots, k} \mu_{A_{j}}(x) \\
X^{0}=\underset{X \in L}{\arg \max } \min _{j=1, \ldots, k} \mu_{A_{j}}(x)
\end{gathered}
$$

To gain (Eq. 6), it is necessary to create membership functions $\mu_{A_{j}(X)} ; j=1, \ldots, k$, reverberating a level of meeting its "own" optimum by the relevant $F_{j}(X), X \in L ; j=1, \ldots, k$. This is contented by the executing of the membership functions [62]. The context of the issue plays an important role in choosing the membership function of objectives and restrictions, whether linear or nonlinear limitation exists. Thanks to this point, one of the feasible fuzzy convolution themes is illustrated as follows [62].

The primary estimation for the $X$-vector is selected. With the aim of scalar minimization/maximization, the minimum/maximum values for each standard $F_{j}(X)$ are formed. Outcomes stand for "ideal" data points $\left\{X_{j}^{0}, j=1, \ldots, m\right\}$.

Consequently, the matrix table $\{T\}$, where the diagonal components are "ideal" data points, is constructed as below:

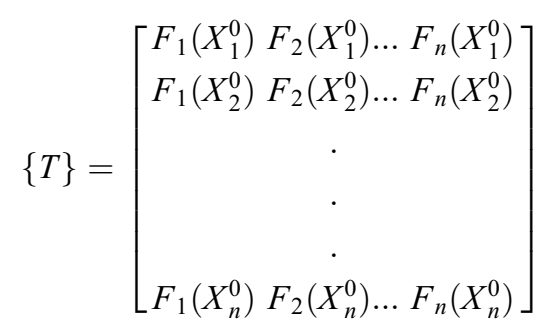

Minimum and maximum limitations for the norms are expressed

$$
\begin{aligned}
& F_{i}^{\min }=\min _{j} F_{j}\left(X_{j}^{0}\right), \quad i=1, \ldots, n \\
& F_{i}^{\max }=\max _{j} F_{j}\left(X_{j}^{0}\right), \quad i=1, \ldots, n
\end{aligned}
$$

Following membership functions are presumed throughout applying fuzzy technique on the generated results For minimized objective functions:

$$
\mu_{F i}(X)= \begin{cases}0 & \text { if } F_{i}(x)>F_{i}^{\max } \\ \frac{F_{i}^{\max }-F_{i}}{F_{i}^{\max }-F_{i}^{\min }} & \text { if } F_{i}^{\min }<F_{i} \leq F_{i}^{\max } \\ 1 & \text { if } F_{i}(x) \leq F_{i}^{\min }\end{cases}
$$


For maximized objective functions:

$$
\mu_{F i}(X)= \begin{cases}1 & \text { if } F_{i}(x)>F_{i}^{\max } \\ \frac{F_{i}-F_{i}^{\min }}{F_{i}^{\max }-F_{i}^{\min }} & \text { if } F_{i}^{\min }<F_{i} \leq F_{i}^{\max } \\ 0 & \text { if } F_{i}(x) \leq F_{i}^{\min }\end{cases}
$$

Limitations of fuzzy are expressed as:

$$
G_{j}(X) \leq G_{j}^{\max }+d_{j}, \quad j=1,2, \ldots, k
$$

In which $d_{j}$ stands for a subjective variable that represents an interval between the accessible shift for the bound $G_{j}^{\max }$ of the $j$ th limitation. Relevant membership functions are formulated as below [10, 11]:

$$
\mu_{G i}(X)= \begin{cases}0 & \text { if } G_{i}(x)>G_{i}^{\max } \\ 1-\frac{G_{j}(x)-G_{j}^{\max }}{d_{j}} & \text { if } G_{i}^{\max }<G_{i}(X) \leq G_{i}^{\max }+d_{j} \\ 1 & \text { if } G_{i}(x) \leq G_{i}^{\max }\end{cases}
$$

As noted previously, the junction between all of fuzzy criteria and restrictions represents the final decision, whereas it is illustrated with the aim of membership function. This issue is lessened to the standard nonlinear programming issues: to determine such values of $X$ and $k$ which maximize $k$ regarding:

$$
\begin{aligned}
& \lambda \leq \mu_{F_{i}}, \quad i=1,2, \ldots, n \\
& \lambda \leq \mu_{G_{j}}, \quad j=1,2, \ldots, k
\end{aligned}
$$

The route of the multi-index issue reveals the sense of the optimality factor and its affinity on the decision-makers' expertise and grasp of the issue.

\section{A.2. LINMAP Decision-Making Method}

An ideal point on the Pareto frontier defined as the point where each objective is optimized irrespective of assuring of other objectives. Obviously, each objective in the multi-criteria optimization does not have the same value as gained by single-objective optimization. Hence, the ideal point is not posited on the Pareto frontier. Throughout the LINMAP approach, with Euclidian non-dimensionalization of all objectives, the interval of each route on the Pareto frontier from the ideal point represented by $d_{i+}$ is calculated as follows:

$$
d_{i+}=\sqrt{\sum_{j=1}^{n} F_{i j}-F_{j}^{\text {Ideal }}}
$$

In which $n$ represents the number of objectives, whereas $i$ represents each route on the Pareto frontier $(i=1,2, \ldots, m)$. In Equation (14), $F_{j}^{\text {Ideal }}$ denotes the ideal value for the $j$ th objective gained by a single-objective optimization. Throughout the LINMAP approach, the outcome with a minimum interval from ideal point is chosen as an ultimate optimum route; meanwhile, $i_{\text {final }}$ represents the $i$ index of an ultimate route:

$$
i_{\text {final }}=i \in \min \left(d_{i+}\right) \quad i=1,2, \ldots, m
$$

\section{A.3. TOPSIS Decision-Making Method}

In addition to the definition of the ideal point, throughout the TOPSIS approach, a non-ideal point should be described and specified. Thanks to this fact, the non-ideal point is defined as the latitude in the objectives region where 
each objective has its arrant amount. Thus, alongside the distance between the outcome and ideal point, $d_{i+}$, the distance from the non-ideal point which represented by $d_{i-}$ is executed as another screening parameter for choosing the ultimate route. Therefore $[10,11]$ :

$$
d_{i-}=\sqrt{\sum\left(F_{i j}-F_{j}^{\text {non-ideal }}\right)^{2}}
$$

Furthering the TOPSIS approach, the $C l_{i}$ variable is formulated as follows:

$$
C l_{i}=\frac{d_{i-}}{d_{i+}+d_{i-}}
$$

Consequently, the final optimum solution is chosen on the basis of minimum $C l_{i}$, so, if $i_{\text {final }}$ denotes the index for the ultimate chosen route, the following expression is true:

$$
i_{\text {final }}=i \in \max \left(C l_{i}\right) ; \quad i=1,2, \ldots, m
$$

